\title{
On the Influence of Extracellular Fluid Volume Expansion and of Uremia on Bicarbonate Reabsorption in Man
}

\author{
Eduardo Slatopolsky, Phillip Hoffsten, Mabel Purkerson, and \\ NeAl S. Bricker \\ From the Renal Division, Department of Internal Medicine, Washington \\ University School of Medicine, St. Louis, Missouri 63110
}

A в S T R A C T The patterns of bicarbonate reabsorption during increasing plasma concentrations were studied in subjects with a range of glomerular filtration rates (GFR) from 170 to $2 \mathrm{ml} / \mathrm{min}$. In a group of five subjects with GFR values above $30 \mathrm{ml} / \mathrm{min}$, paired bicarbonate titration studies were performed first under conditions which minimized extracellular fluid (ECF) volume expansion, and second under conditions which were conducive to exaggerated expansion of ECF volume. In patients with GFR values below $30 \mathrm{ml} / \mathrm{min}$, a single protocol was employed. Studies also were performed on two patients with far advanced renal disease who were nephrotic and exhibited a sodium-retaining state. When ECF volume expansion was minimized in the nonuremic subjects, values for bicarbonate reabsorption were well in excess of the usually accepted Tm level and over the range of plasma bicarbonate concentrations employed, no evidence of a Tm phenomenon was observed. A similar pattern emerged in the two nephrotic patients despite the presence of uremia. However, with both exaggerated expansion of ECF volume (GFR greater than 30 ) and in patients with advanced renal disease in the absence of exaggerated ECF volume expansion a tendency towards saturation kinetics for bicarbonate reabsorption was demonstrable. In comparing the minimized with the exaggerated expansion studies, evidence emerged for a decrease in both bicarbonate reabsorption per unit of GFR and the absolute rate of bicarbonate reabsorption. When ECF volume expansion was exaggerated in uremic patients after stable rates of bicarbonate reabsorption had been achieved, a decrease in reabsorption per unit of GFR and in absolute bicarbonate reabsorption occurred. The possible relationship

This work was presented in part at the 2nd Annual Meeting of the American Society of Nephrology, Washington, D. C. An abstract has been published: 1968 Proceedings of the American Society of Nephrology. 2: 62.

Received for publication 27 August 1969 and in revised form 19 November 1969. of the factors controlling sodium excretion to the observed patterns of bicarbonate reabsorption is considered in the text.

\section{INTRODUCTION}

It is generally accepted that in normal man the kinetics of bicarbonate reabsorption by the kidney are characterized by an apparent $\mathrm{Tm}$ such that a maximum of approximately $2.8 \mathrm{mEq}$ of bicarbonate may be reabsorbed per $100 \mathrm{ml}$ of GFR $(1,2)$. Moreover, in uremia, Roberts, Randall, Vanamee, and Poppell (3) reported a Tm value for bicarbonate that fell within the normal range. Unfortunately the standard technique for performing a bicarbonate titration experiment involves marked expansion of extracellular fluid (ECF) volume; volume expansion is characteristically associated with a decrease in fractional sodium reabsorption and with an increase in natriuresis per nephron. Furthermore, a decrease in fractional sodium reabsorption and an increase in sodium excretion rate per nephron also are characteristic of the uremic state. On a priori grounds, therefore, the expansion of ECF volume in normal subjects produced by the bicarbonate titration technique and nephron reduction in uremic patients might influence bicarbonate reabsorption through a primary effect on sodium transport; a regulatory and homeostatic change in the patterns of sodium excretion thus could lead to a change in bicarbonate reabsorption that might be fortuitous rather than regulatory or homeostatic. It is of interest in this context that recent studies in the normal rat have shown that when special precautions are taken to minimize ECF volume expansion during the performance of bicarbonate titration studies, no $\mathrm{Tm}$ is demonstrable over a wide range of plasma bicarbonate concentrations (4). However, when ECF volume expansion was exaggerated, a definite tendency for bicarbonate reabsorption to become constant was evident (4). Moreover, a transformation in the bicarbonate titration curve similar to 
that evoked by volume expansion has been seen in rats subjected to marked reduction in total nephron population (5).

The present studies were performed to reevaluate the patterns of bicarbonate reabsorption in man with particular reference to the influence of extracellular fluid volume expansion and of uremia. In a group of nonuremic subjects, titration studies were performed under conditions of "minimized" and "exaggerated" ECF volume expansion. Titration studies also were performed in a group of patients with moderately to far-advanced bilateral renal disease. In three patients with uremia, the effects of exaggerated ECF volume expansion were measured after the apparent bicarbonate $\mathrm{Tm}$ had been reached. Finally, the kinetics of bicarbonate reabsorption were studied in two patients with advanced renal disease who were nephrotic and thus did not exhibit the typical natriuresis per nephron of uremia.

\section{METHODS}

A total of 31 studies was performed on 17 subjects. Two were normal adult voluntcers, 15 were patients with chronic bilateral renal disease of varying severity. Nine of the patients had chronic glomerulonephritis; four, pyelonephritis; one nephrosclerosis; and one, congenital hypoplastic kidneys. Two of the patients with glomerulonephritis and low glomerular filtration rates were nephrotic at the time of study and their results will be considered separately. The total group studied provided a range of glomerular filtration rates from $170 \mathrm{ml} / \mathrm{min}$ to $2 \mathrm{ml} / \mathrm{min}$. In five subjects with GFR values above $30 \mathrm{ml} / \mathrm{min}$, two different protocols were employed in the performance of bicarbonate titration studies. One was designed to minimize ECF volume expansion, the other to allow exaggerated expansion of the ECF. In the nephrotic patients and in the majority of patients with GFR's below $30 \mathrm{ml} / \mathrm{min}$, exaggerated ECF expansion was not induced and only a single standard protocol for bicarbonate infusion was employed. However, in three nonnephrotic patients with low glomerular filtration rates, the effects of ECF expansion were observed on the maximal rate of bicarbonate reabsorption.

In order to decrease plasma bicarbonate concentrations to subnormal levels, from 2 to $8 \mathrm{~g}$ of ammonium chloride were given orally for 3-5 days before study in many of the subjects. The dose was prorated on the basis of the GFR and the steady-state plasma bicarbonate concentration. To eliminate mineralocorticoid insufficiency as an indeterminate variable, all subjects received deoxycorticosterone acetate (DOCA), $10 \mathrm{mg}$ intramuscularly, on the morning of study. To avoid potassium depletion during titration studies, supplementary $\mathrm{KCl}$ was administered in most instances.

The specific protocols that were employed were as follows.

\section{Subjects with GFR values greater than $30 \mathrm{ml} / \mathrm{min}$}

Minimized expansion. Dietary intake of salt was restricted to $1 \mathrm{~g} /$ day for $1 \mathrm{wk}$ before study. In some subjects, furosemide (40-120 mg/day) was given orally for several days in order to decrease extracellular fluid volume in advance of the titration studies. This drug was discontinued $48 \mathrm{hr}$ before the studies were performed. If plasma potas- sium concentrations fell during the period of diuretic administration, supplementary potassium was administered. During a 60-90 min equilibration period and the first three clearance periods, no bicarbonate was infused; the inulin used to maintain constant plasma inulin concentrations was administered in a $5 \%$ dextrose and water solution at a rate of 2.5 $\mathrm{ml} / \mathrm{min}$. Thereafter the infusion was changed to a $5 \%$ sodium bicarbonate solution which was delivered at a rate of $2-2.5 \mathrm{ml} / \mathrm{min}$.

Exaggerated expansion. Dietary intake of salt was maintained between 12 and $16 \mathrm{~g} /$ day for 1 wk before study. 9 - $\alpha$-fluorohydrocortisone in a dose of $0.1 \mathrm{mg}$ was administered twice daily during this period. The composition of the infusion during the equilibration period and three control clearance periods was the same as that used in the minimized expansion studies. After the third clearance period the infusion was changed to a $2-2.5 \%$ sodium bicarbonate solution which was administered at a rate of $6-12 \mathrm{ml} / \mathrm{min}$.

Nonnephrotic patients with GFR values less than $30 \mathrm{ml} / \mathrm{min}$

These patients were maintained on a $4 \mathrm{~g}$ salt diet for at least 5 days before study and each was in external salt balance at the time of study with neither evident volume depletion nor edema. The infusion procedure employed in this group of patients was the same as that used in the minimized expansion group.

\section{Nephrotic patients}

Two patients with far-advanced renal disease (GFR 7 and $17 \mathrm{ml} / \mathrm{min}$ respectively) had marked proteinuria and hypoalbuminemia and both were in positive sodium balance on a $1 \mathrm{~g}$ salt diet despite their uremic state. Thus neither of these two patients exhibited the natriuresis per nephron that characterizes the great majority of patients with comparable levels of glomerular filtration rate. Both patients were maintained on a $1 \mathrm{~g}$ salt diet for 1 wk before study and both were edematous at the time of the titration studies.

Glomerular filtration rate was measured using a constant infusion of inulin. From 10 to 25 clearance periods were obtained in individual studies and each period varied in duration from 10 to $35 \mathrm{~min}$. Urine was collected using an in-lying Foley catheter. Special precautions were taken to maintain sterility (6). These included the use of an antibiotic ointment for lubricating the catheter and rinsing of the bladder with a neomycin solution at the conclusion of the study. In all subjects, urine cultures were obtained on one or more occasions after the conclusion of each study and in no instance was a positive culture obtained. Urine was collected under oil in acid-washed tubes. Neither water nor air rinses were used in order to avoid introducing changes in the urinary $\mathrm{PCO}_{2}$, but pressure was applied to the bladder routinely at the end of each collection. Blood was collected from a catheter in the radial artery continuously throughout each clearance period in order to obtain integrated plasma bicarbonate concentrations. The blood was collected into heparinized tubes under mineral oil. Informed consent was obtained from each subject employed in these studies.

Blood and urine $\mathrm{pH}$ and $\mathrm{PCO}_{2}$ values were determined immediately after collection of the samples and plasma bicarbonate concentrations were estimated as the study proceeded. In this manner the rate and degree of rise of plasma bicarbonate could be monitored and controlled. Determinations of $\mathrm{pH}$ and $\mathrm{PCO}_{3}$ on both blood and urine were made 
TABLE I

A Bicarbonate Titration Study on a Normal Volunteer during Minimal ECF Volume Expansion

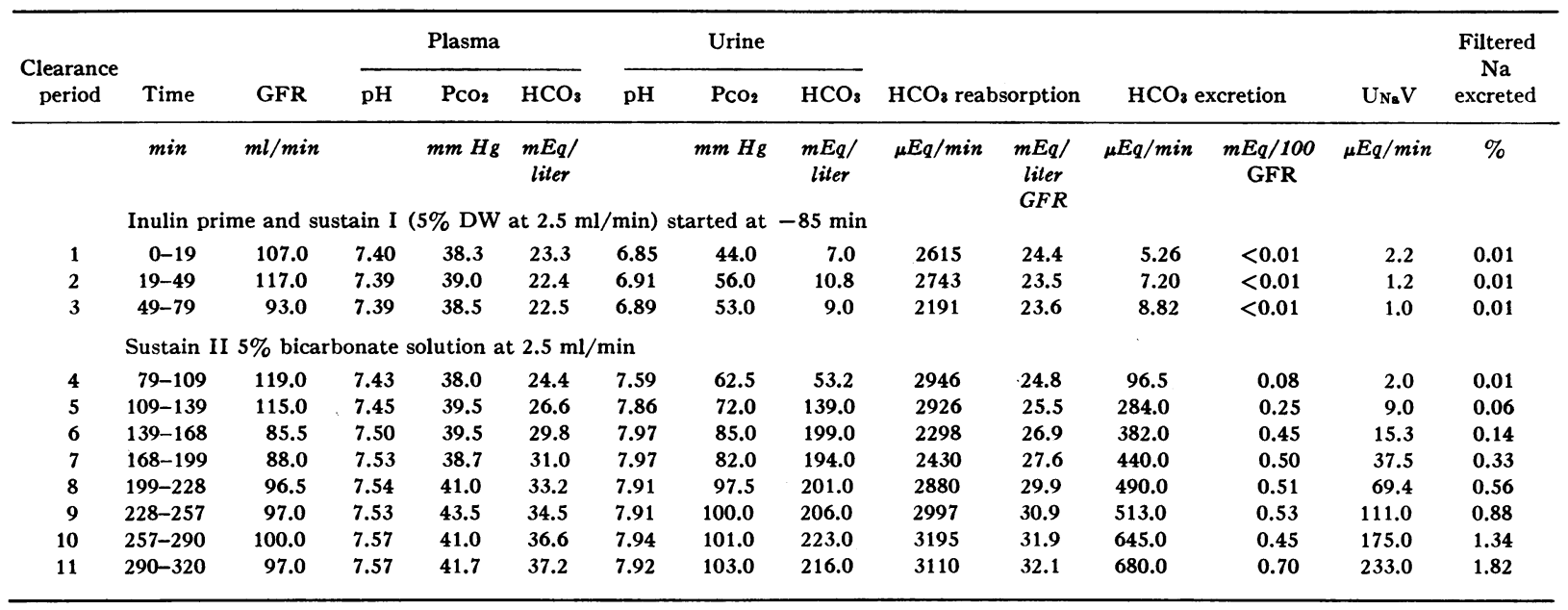

$\mathrm{U}_{\mathrm{NaV}}=$ sodium excretion rate. Donnan correction (1.05) was applied in calculating the filtered load of bicarbonate.

using an Instrumentation Laboratory Inc. microgas analyzer (model IL 113-SL) and a Medtron $\mathrm{pH}$ meter (model 27). Bicarbonate concentrations in urine and plasma were calculated using the Henderson-Hasselbalch equation with a $\mathrm{pK}^{\prime}$ value of 6.1 and a solubility coefficient of 0.0301 for plasma. For urine, the $\mathrm{pK}^{\prime}$ value was calculated using the formula $\mathrm{pK}^{\prime}=6.33-0.5 \sqrt{\mathrm{B}}$, where $\mathrm{B}$ represents the sum of $\mathrm{Na}+\mathrm{K}$ concentrations. An alpha value of 0.0309 for urine was used. Inulin was determined according to the method of Roe, Epstein, and Goldstein (7). Plasma and urine sodium and potassium concentrations were measured with an internally compensated flame photometer.

\section{RESULTS}

Subjects with GFR values greater than $30 \mathrm{ml} / \mathrm{min}$. The results of a bicarbonate titration study in a normal subject in whom extracellular fluid volume expansion was minimized are shown in Table I. The plasma bicarbonate concentration was increased progressively from an average value of $22.7 \mathrm{mEq} /$ liter in the three control periods to a maximum value of $37.2 \mathrm{mEq} /$ liter in the final period of study. No tendency for bicarbonate reabsorption to plateau at the usually accepted value of $2.7-2.8 \mathrm{mEq} / 100 \mathrm{ml}$ of GFR was observed. Indeed, during the final clearance period, the value for bicarbonate reabsorption was $3.21 \mathrm{mEq} / 100 \mathrm{ml}$ of GFR $(32.1 \mathrm{mEq} /$ liter GFR). The same subject was restudied using the protocol for exaggerated expansion of ECF described in the Methods section. The results are shown in Table II. Plasma bicarbonate concentration averaged 18.6

TABLE II

A Bicarbonate Titration Study during Exaggerated ECF Expansion on the Same Subject Depicted in Table I

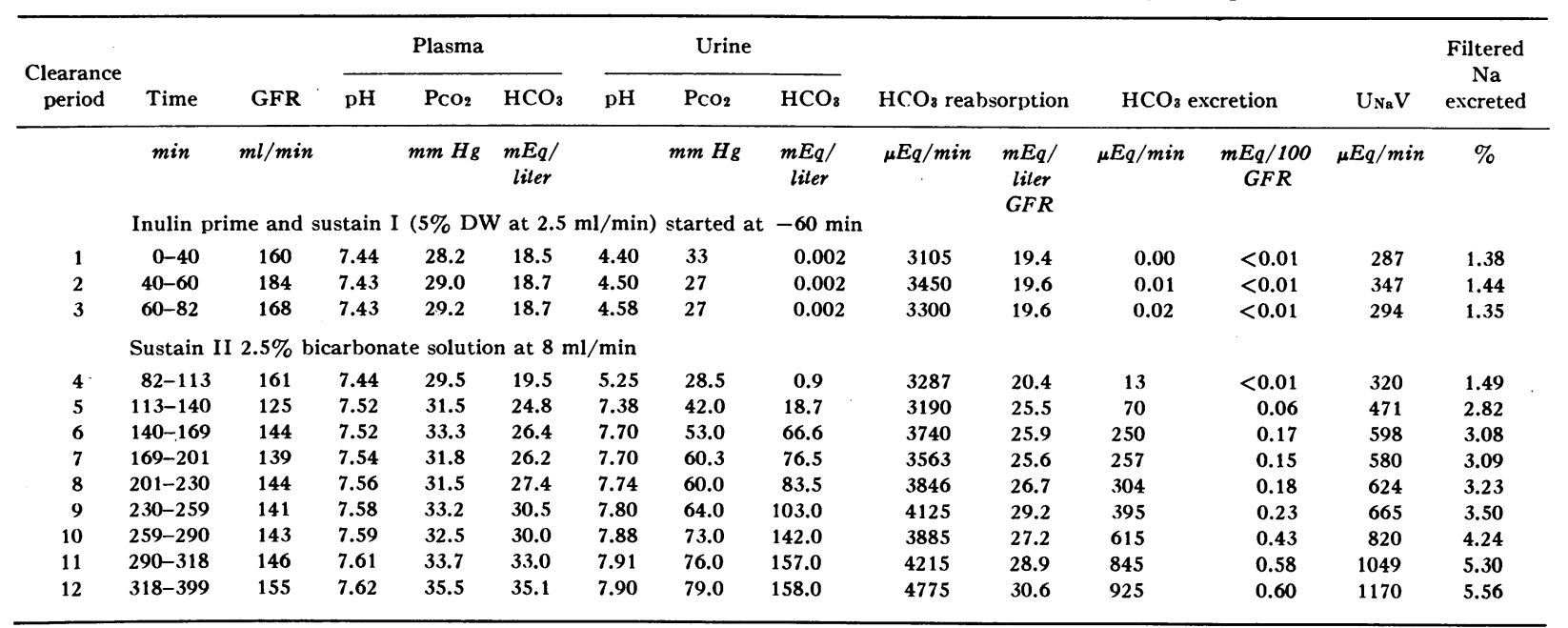


$\mathrm{mEq} /$ liter during the three control periods. Levels were elevated thereafter in a progressive manner to a final value of $35.1 \mathrm{mEq} /$ liter during the 12 th clearance period. Bicarbonate reabsorption averaged $19.5 \mathrm{mEq} /$ liter GFR during the control periods. Values then increased to $29.2 \mathrm{mEq} /$ liter GFR in the 9th clearance period but a tendency for stabilization was observed during the final four clearance periods when plasma bicarbonate concentrations ranged from 30.5 to $35.1 \mathrm{mEq} /$ liter. It is of interest that even in the presence of exaggerated expansion of ECF and with GFR markedly increased in relation to the initial study, values for reabsorption approximated $30 \mathrm{mEq} /$ liter $\mathrm{GFR}$ rather than 27 to 28 $\mathrm{mEq} /$ liter GFR.

With minimized expansion (Table I) sodium excretion was negligible during the control periods, reflecting the prior regimen of sodium deprivation and diuretic administration, and the maximum rate of sodium excretion observed was $233 \mu \mathrm{Eq} / \mathrm{min}$ (1.82\% of the filtered sodium) during the final clearance period when the bicarbonate excretion was $680 \mu \mathrm{Eq} / \mathrm{min}$. In the second study with exaggerated expansion of ECF volume ( Table II) sodium excretion rate averaged $309 \mu \mathrm{Eq} / \mathrm{min}$ during the control clearance periods and the values increased progressively thereafter to a maximum rate of $1170 \mu \mathrm{Eq} / \mathrm{min}$ ( $5.56 \%$ of the filtered sodium). Due to the greater GFR in the second study, the absolute rates of reabsorption of both bicarbonate and sodium were higher during exaggerated than minimized expansion.

The results in the second normal subject are presented in Table III and IV. With minimized expansion of ECF
(Table III) plasma bicarbonate concentrations were increased from a control value of $20.4 \mathrm{mEq} /$ liter to a peak value of $32.7 \mathrm{mEq} /$ liter. Bicarbonate reabsorption increased throughout the study showing no evidence of stabilization at a constant level and at the peak plasma bicarbonate concentration, the value for reabsorption was $30.7 \mathrm{mEq} /$ liter GFR. During exaggerated expansion of ECF volume (Table IV) the plasma bicarbonate concentration was increased from the control value of $15.4 \mathrm{mEq} /$ liter to a maximum value of 35.6 $\mathrm{mEq} /$ liter. Bicarbonate reabsorption stabilized between a range of 26.1 and $27.5 \mathrm{mEq} /$ liter GFR during the last seven clearance periods corresponding to a plasma bicarbonate concentration range of $28.9-35.6 \mathrm{mEq} /$ liter. Sodium excretion patterns also differed markedly in the two studies. With minimized ECF volume expansion the maximal rate of sodium excretion observed at the peak plasma bicarbonate concentration was $239 \mu \mathrm{Eq} / \mathrm{min}$ or $1.23 \%$ of the filtered sodium. At the same plasma bicarbonate concentration in the presence of exaggerated expansion (period 16), the comparable values were $709 \mu \mathrm{Eq} / \mathrm{min}$ and $4.45 \%$ of the filtered sodium excreted. The average value for glomerular filtration rate was greater during the exaggerated expansion than the minimized expansion study; however, above a plasma bicarbonate concentration of $28 \mathrm{mEq} /$ liter the difference disappeared and, in fact, values were higher in the minimized expansion study. Consequently, the absolute rate of bicarbonate reabsorption (as well as the rate of reabsorption per unit of GFR) was greater in the minimized expansion study.

TABLE III

A Bicarbonate Titration Study on a Normal Volunteer during Minimal ECF Volume Expansion

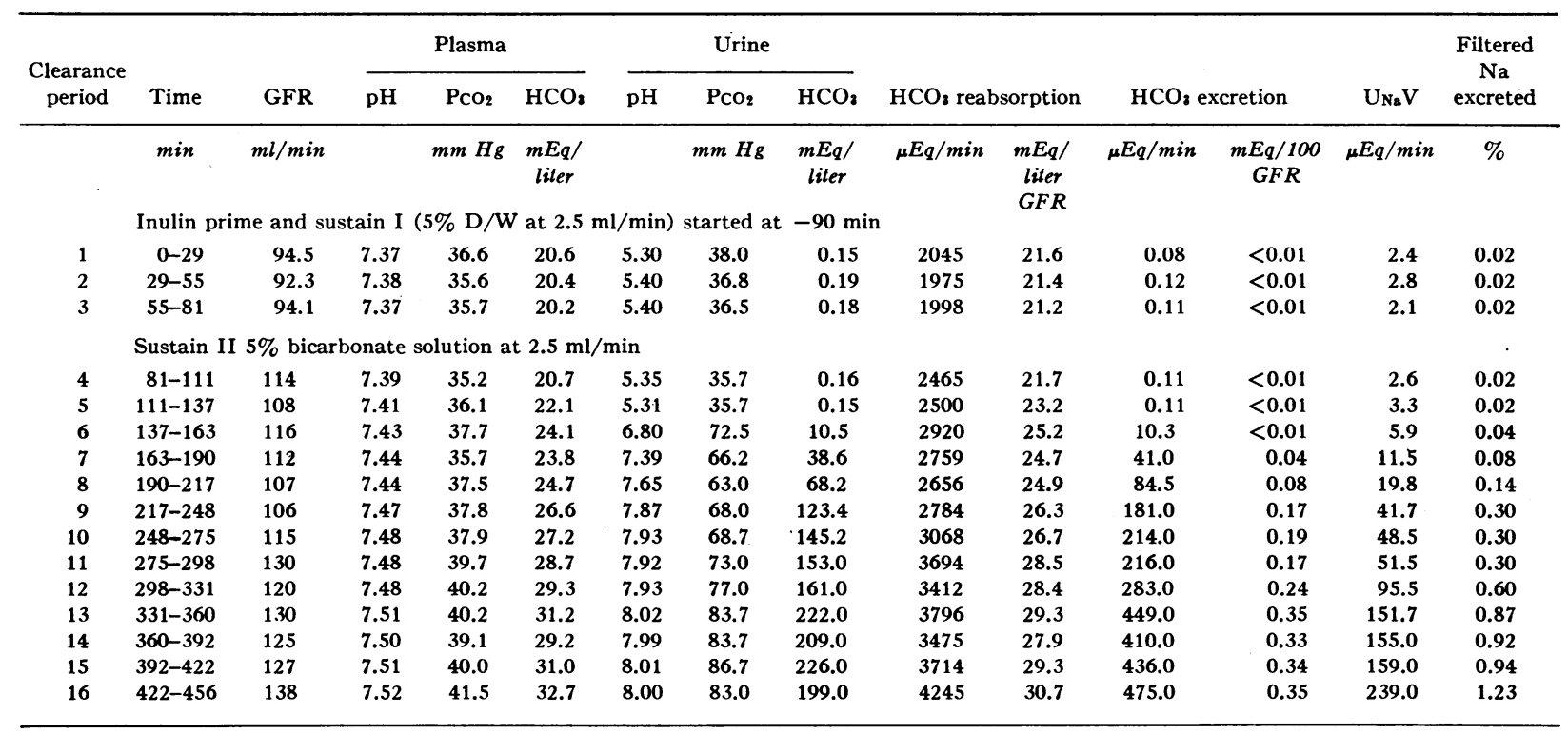


TABLE IV

A Bicarbonate Titration Study during Exaggerated ECF Expansion in the Same Subject Depicted in Table III

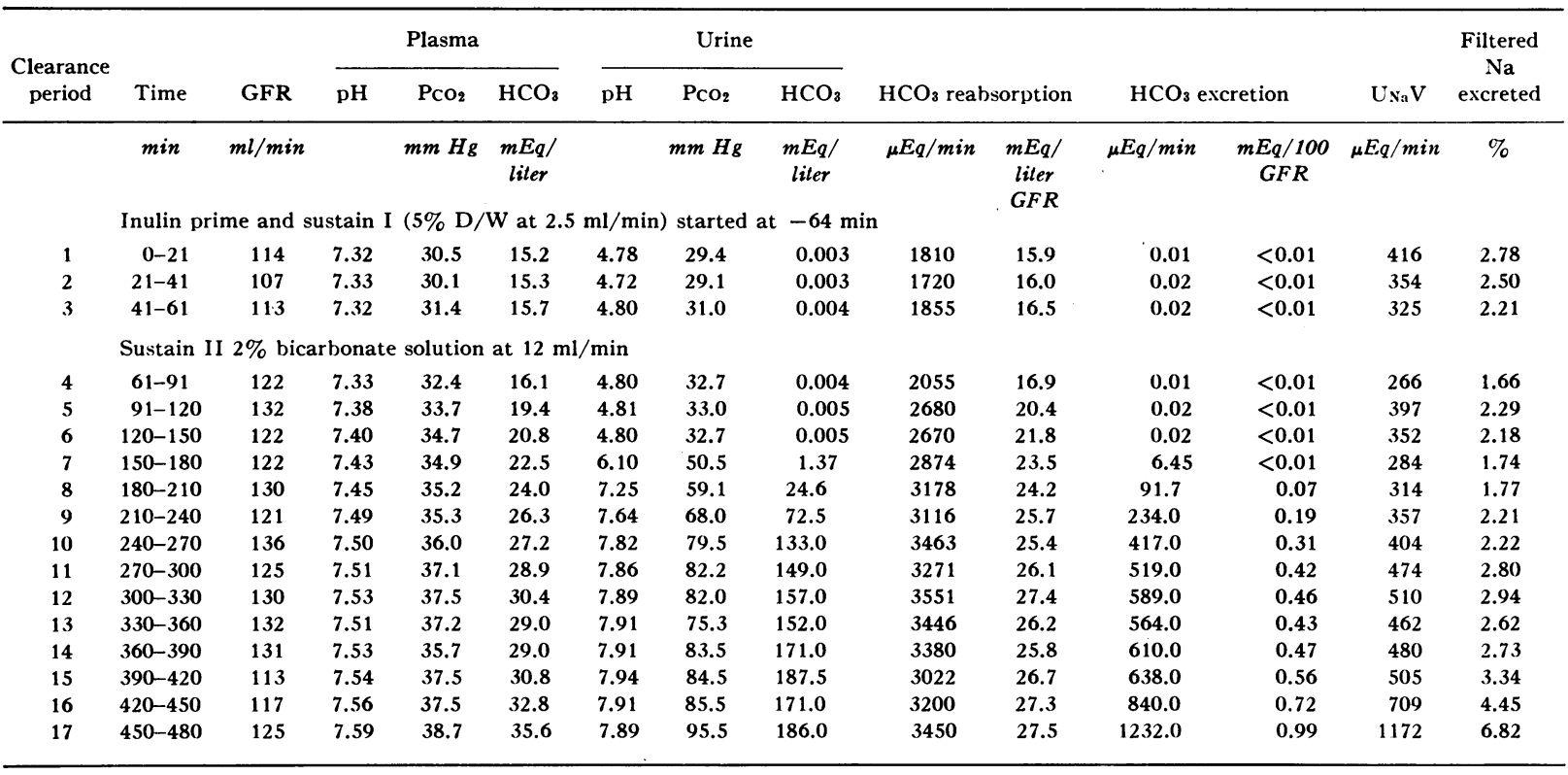

Similar paired studies were performed on three patients with bilateral renal disease. Glomerular filtration rates for these patients averaged $133 \mathrm{ml} / \mathrm{min}$ (this pa-

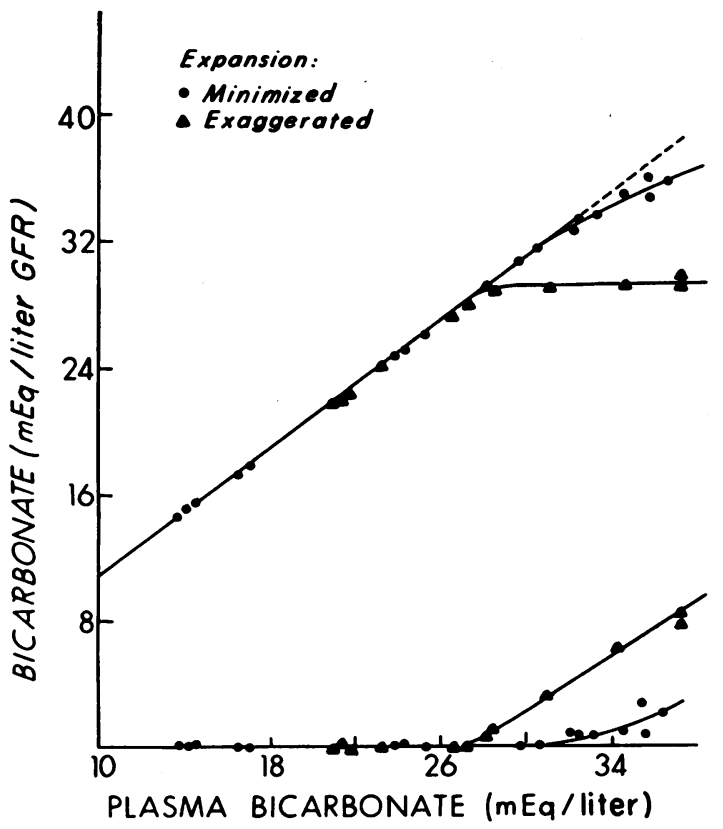

Figure 1 Bircarbonate titration curves obtained from a patient with a GFR of $37 \mathrm{ml} / \mathrm{min}$ studied under conditions of minimized and exaggerated expansion of ECF volume. The two upper curves depict bicarbonate reabsorption, the bottom curves bicarbonate excretion. tient had clinical and histologic evidence of chronic glomerulonephritis despite the high GFR), $80 \mathrm{ml} / \mathrm{min}$. (bilateral pyelonephritis), and $37 \mathrm{ml} / \mathrm{min}$ (bilateral pyelonephritis). In the minimized expansion studies, plasma bicarbonate levels were elevated to peak values of $39.3,29.8$, and $36.5 \mathrm{mEq} /$ liter respectively. But in none of the patients was a Tm observed. However when volume expansion was exaggerated the titration curves were different in each instance and reabsorption tended to stabilize at values of $35.2,28.2$, and $28.2 \mathrm{mEq} /$ liter. The two titration curves for the patient with a GFR of $37 \mathrm{ml} / \mathrm{min}$ are shown in Fig. 1 .

In three of the five sets of paired studies, values for GFR and plasma bicarbonate concentrations were closely comparable during minimized expansion and exaggerated expansion of ECF in three or more consecutive clearance periods. Thus the opportunity exists to examine absolute values for bicarbonate reabsorption at the same filtered load of bicarbonate in the same subjects under different conditions of volume expansion. The data are shown in Table V. In subject 2, six consecutive clearance periods obtained during minimized expansion are compared with eight consecutive periods obtained during exaggerated expansion. GFR averaged 128 vs. $126 \mathrm{ml} / \mathrm{min}$ respectively in the two studies. Plasma bicarbonate concentrations averaged 30.4 vs. $30.5 \mathrm{mEq} /$ liter. Filtered load of bicarbonate ranged from 3695 to $4720 \mu \mathrm{Eq} / \mathrm{min}$ (mean 4101) in the minimized expansion study and from 3660 to 4680 (mean 4024) in the exaggerated expansion study. Absolute bicarbonate reabsorption aver- 
TABLE V

Values for GFR, Plasma Bicarbonate Concentrations, Bicarbonate Reabsorption, and Excretion in the Same Subjects during Minimized vs. Exaggerated Expansion of ECF Volume

\begin{tabular}{|c|c|c|c|c|c|c|c|c|c|c|}
\hline & \multicolumn{5}{|c|}{ Minimized expansion } & \multicolumn{5}{|c|}{ Exaggerated expansion } \\
\hline & GFR & $\begin{array}{c}\text { Plasma } \\
\mathrm{HCO}_{z}\end{array}$ & $\begin{array}{l}\text { Filtered } \\
\text { load } \\
\mathrm{HCO}_{3}\end{array}$ & $\begin{array}{l}\text { Bicarbonate } \\
\text { excretion }\end{array}$ & $\begin{array}{l}\text { Bicarbonate } \\
\text { reabsorption }\end{array}$ & GFR & $\begin{array}{c}\text { Plasma } \\
\mathrm{HCO}_{3}\end{array}$ & $\begin{array}{c}\text { Filtered } \\
\text { load } \\
\mathrm{HCO}_{3}\end{array}$ & $\begin{array}{l}\text { Bicarbonate } \\
\text { excretion }\end{array}$ & $\begin{array}{l}\text { Bicarbonate } \\
\text { reabsorption }\end{array}$ \\
\hline & $m l / m i n$ & $m E q /$ liter & $\mu E q / \min$ & $\mu E q / \min$ & $\mu E q / \min$ & $m l / m i n$ & $m E q /$ liter & ${ }_{a} E q / \min$ & $\mu E q / \min$ & $\mu E q / \min$ \\
\hline \multirow{8}{*}{ Subject 2} & 130 & 28.7 & 3910 & 216 & 3694 & 136 & 27.2 & 3880 & 417 & 3463 \\
\hline & 120 & 29.3 & 3695 & 283 & 3412 & 125 & 28.9 & 3790 & 519 & 3271 \\
\hline & 130 & 31.2 & 4245 & 449 & 3796 & 130 & 30.4 & 4140 & 589 & 3551 \\
\hline & 125 & 29.2 & 3885 & 410 & 3475 & 132 & 29.0 & 4010 & 564 & 3446 \\
\hline & 127 & 31.0 & 4150 & 436 & 3714 & 131 & 29.0 & 3990 & 610 & 3380 \\
\hline & 138 & 32.7 & 4720 & 475 & 4245 & 113 & 30.8 & 3660 & 638 & 3022 \\
\hline & & & & & & 117 & 32.8 & 4040 & 840 & 3200 \\
\hline & & & & & & 125 & 35.6 & 4680 & 1232 & 3448 \\
\hline Mean & 128 & 30.4 & 4101 & 378 & 3723 & 126 & 30.5 & 4024 & 676 & 3348 \\
\hline \multirow[t]{10}{*}{ Subject 3} & & & & & & & & & & \\
\hline & 38.6 & 29.7 & 1203 & 1 & 1202 & 42.5 & 28.2 & 1260 & 19 & 1241 \\
\hline & 40.5 & 32.3 & 1374 & 1 & 1373 & 38.1 & 28.3 & 1132 & 34 & 1098 \\
\hline & 39.8 & 30.6 & 1277 & 2 & 1275 & 40.6 & 31.0 & 1322 & 131 & 1191 \\
\hline & 35.2 & 33.2 & 1226 & 26 & 1200 & 41.8 & 34.4 & 1509 & 274 & 12.35 \\
\hline & 35.7 & 32.1 & 1204 & 34 & 1170 & 42.4 & 37.4 & 1665 & 376 & 1289 \\
\hline & 38.6 & 35.7 & 1449 & 36 & 1413 & & & & & \\
\hline & 38.0 & 34.6 & 1379 & 43 & 1336 & & & & & \\
\hline & 35.4 & 36.5 & 1358 & 85 & 1273 & & & & & \\
\hline & 42.6 & 35.4 & 1584 & 138 & 1446 & & & & & \\
\hline Mean & 38.3 & 33.3 & 1340 & 41 & 1299 & 41.1 & 31.9 & 1378 & 167 & 1211 \\
\hline \multirow[t]{5}{*}{ Subject 4} & & & & & & & & & & \\
\hline & 152 & 29.4 & 4700 & 57 & 4644 & 160 & 29.4 & 4990 & 451 & 4539 \\
\hline & 152 & 30.3 & 4840 & 174 & 4666 & 176 & 30.7 & 5680 & 469 & 5211 \\
\hline & 163 & 30.9 & 5290 & 268 & 5022 & 143 & 32.5 & 4880 & 734 & 4146 \\
\hline & 149 & 33.3 & 5210 & 310 & 4900 & & & & & \\
\hline Mean & 154 & 31.0 & 5010 & 202 & 4808 & 160 & 30.9 & 5184 & 551 & 4633 \\
\hline
\end{tabular}

Using the $t$ test (method of set comparison) there is no significant difference between the filtered loads of bicarbonate in minimized vs. exaggerated expansion in any of the three subjects. However bicarbonate excretion is significantly greater $(0.05>P>0.02)$ during exaggerated expansion in all three subjects.

In each of the three subjects values for GFR and plasma bicarbonate concentration were closely comparable during three or more consecutive periods.

aged $3723 \mu \mathrm{Eq} / \mathrm{min}$ (range $3412-4245$ ) in the minimized expansion study; the comparable value during exaggerated expansion was $3348 \mu \mathrm{Eq} / \mathrm{min}$ (range 30223463 ). In subject 3 , GFR averaged $38.3 \mathrm{ml} / \mathrm{min}$ during minimized expansion and $41.1 \mathrm{ml} / \mathrm{min}$ during exaggerated expansion. The mean value for the filtered load of bicarbonate was slightly higher during exaggerated expansion, but the absolute value for bicarbonate reabsorption was $1214 \mu \mathrm{Eq} / \mathrm{min}$ during exaggerated expansion and $1299 \mu \mathrm{Eq} / \mathrm{min}$ during minimized expansion. In subject 4 , GFR averaged $154 \mathrm{ml} / \mathrm{min}$ during minimized expansion and $160 \mathrm{ml} / \mathrm{min}$ during exaggerated expansion. The filtered load of bicarbonate averaged $174 \mu \mathrm{Eq} / \mathrm{min}$ less during minimized than exaggerated expansion whereas the mean value for bicarbonate reabsorption was $175 \mu \mathrm{Eq} / \mathrm{min}$ less during exaggerated expansion.

Patients with advanced renal disease. In patients with advanced renal disease a single standard protocol was employed. The patients were not salt deprived before study and the infusion rate for bicarbonate was the same as that used in the minimized expansion studies already described. Fig. 2 depicts the results of a

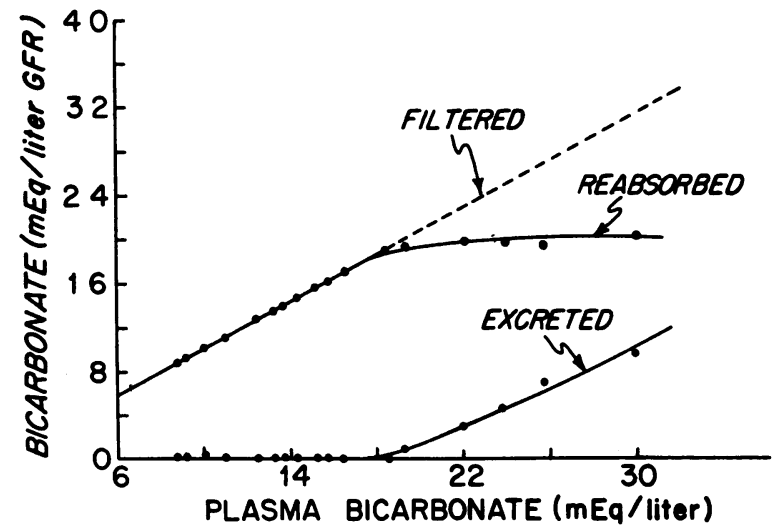

FIGURE 2 Bicarbonate titration curve in a patient with far advanced bilateral renal disease and a glomerular filtration rate of $2 \mathrm{ml} / \mathrm{min}$. 


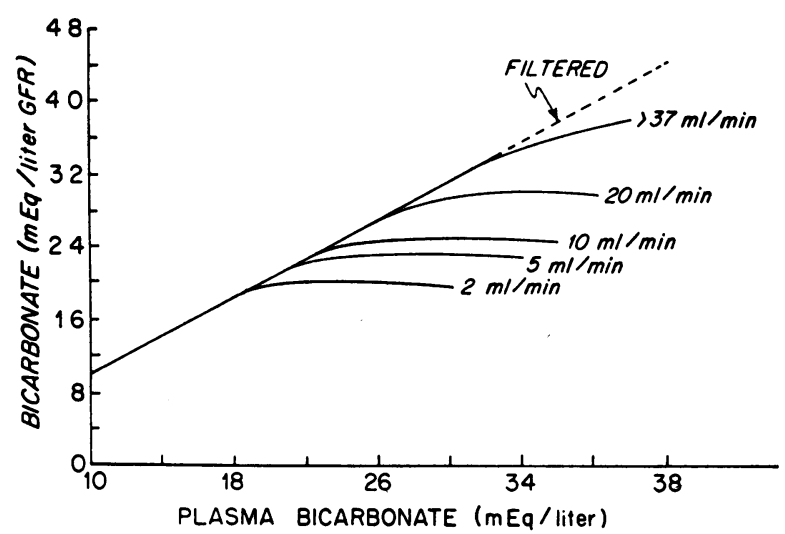

FIGURE 3 Representative bicarbonate titration curves for patients with GFR's ranging from 170 to $2 \mathrm{ml} / \mathrm{min}$. The curve labeled greater than $37 \mathrm{ml} / \mathrm{min}$ is a mean titration curve for five subjects. The other curves are single titration curves but are representative of other patient's with GFR's in the same range.

titration study in a patient with far-advanced chronic glomerulonephritis and a GFR of $2 \mathrm{ml} / \mathrm{min}$. Bicarbonate was excreted into the urine at a plasma bicarbonate concentration of $18 \mathrm{mEq} /$ liter and reabsorption stabilized at a value of $19 \mathrm{mEq} /$ liter GFR. In Fig. 3 a summary is presented of representative titration curves obtained in patients with advanced renal disease at different levels of GFR. With a GFR of $37 \mathrm{ml} / \mathrm{min}$ or greater, no apparent $T m$ was demonstrable when ECF volume expansion was minimized. In the patients with GFR's of $20 \mathrm{ml} / \mathrm{min}$ or lower an apparent $\mathrm{Tm}$ was observed and the lower the value for GFR, the lower the value for the $\mathrm{Tm}$.

The relationship between the fractional excretion of sodium and bicarbonate reabsorption in the group of 12 uremic patients is shown in Fig. 4. The figure was constructed by averaging all values in each patient for bicarbonate reabsorption and fractional sodium excretion at plasma bicarbonate concentrations between 28 and 31 $\mathrm{mEq} /$ liter. It is evident that the greater the rate of fractional sodium excretion, the lower the value for bicarbonate reabsorption.

The effects of acute ECF volume expansion on the bicarbonate Tm in uremic patients. In three uremic patients, ECF volume was expanded after bicarbonate reabsorption had stabilized at an apparent $\mathrm{Tm}$ level. The results in each of the three patients were qualitatively similar and the detailed value for one of the three patients are shown in Table VI. GFR in this patient averaged $10.5 \mathrm{ml} / \mathrm{min}$ during the three control clearance periods. Values for plasma bicarbonate concentrations were increased from a control level of $15.7 \mathrm{mEq} /$ liter to a peak level of approximately $34.9 \mathrm{mEq} / \mathrm{liter}$. Bicarbonate reabsorption stabilized at approximately 25 $\mathrm{mEq} /$ liter GFR. With the superimposition of acute ECF volume expansion, values for reabsorption fell precipitously to a final value of $19.8 \mathrm{mEq} /$ liter GFR. Of interest is the fact that GFR and the filtered load of bicarbonate increased markedly during the period of acute expansion. Yet absolute rates of bicarbonate excretion failed to increase proportionately; indeed in comparing the last four periods of the standard titration procedure with the four periods in which acute volume expansion was superimposed, GFR increased from 12.5 to $13.4 \mathrm{ml} /$ min and the filtered load of bicarbonate increased from 350 to $455 \mu \mathrm{Eq} / \mathrm{min}$, but the absolute rate of bicarbonate reabsorption decreased from 307 to $277 \mu \mathrm{Eq} / \mathrm{min}$.

Nephrotic patients. GFR values in the two nephrotic patients averaged 7 and $17 \mathrm{ml} / \mathrm{min}$. Both were in positive sodium balance on a $1 \mathrm{~g}$ salt intake. The detailed results in the patient with the lower GFR are shown in Table VII. Plasma bicarbonate concentrations were increased from a mean value of $17.6 \mathrm{mEq} /$ liter during the three control periods to a maximum value of $34.5 \mathrm{mEq} /$ liter during the 17 th clearance period. Bicarbonate excretion in the urine remained at negligible levels until the plasma bicarbonate concentration approached 30 $\mathrm{mEq} /$ liter and at the highest plasma level observed, bicarbonate excretion rate was under $10 \mu \mathrm{Eq} / \mathrm{min}(0.12$ $\mathrm{mEq} / 100 \mathrm{ml} \mathrm{GFR})$. Bicarbonate reabsorption increased from an average value of $18.5 \mathrm{mEq} /$ liter GFR in the control periods to a peak value in excess of $35 \mathrm{mEq} /$ liter GFR during the final two clearance periods. Thus, bicarbonate reabsorption was essentially complete throughout the entire study despite the existence of far-advanced renal disease and chemi-

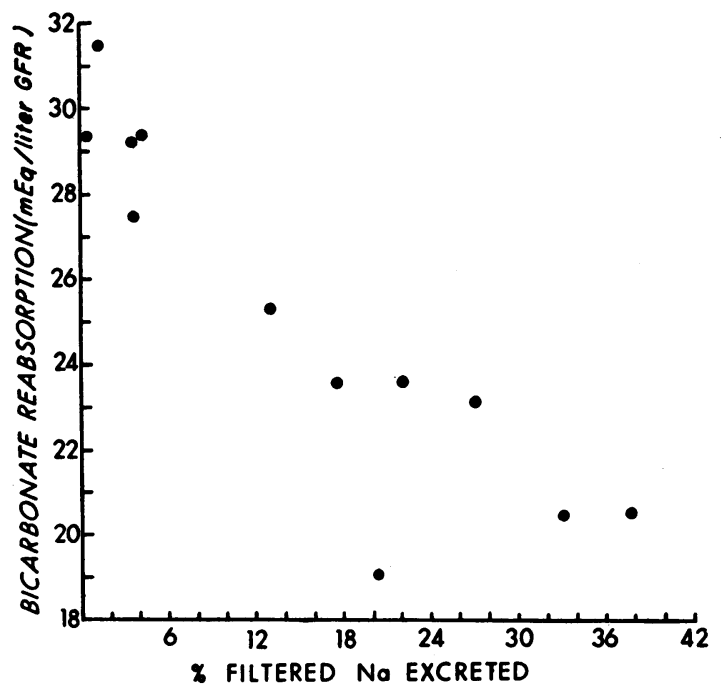

Figure 4 Comparison of bicarbonate reabsorption with fractional sodium excretion in the 12 uremic patients. In each patient all values for bicarbonate reabsorption and fractional sodium excretion were averaged at plasma bicarbonate concentrations between 28 and $31 \mathrm{mEq} /$ liter. 
TABLE VI

The Effects of Exaggerated ECF Volume Expansion on Bicarbonate Reabsorption after an Apparent Tm had been Reached in a Uremic Patient

\begin{tabular}{|c|c|c|c|c|c|c|c|c|}
\hline $\begin{array}{l}\text { Clearance } \\
\text { period }\end{array}$ & GFR & $\mathrm{PHCOs}_{3}$ & F.L. $\mathrm{HCO}_{3}$ & UнсозV & $\mathrm{U}_{\mathrm{Na}} \mathrm{V}$ & $\begin{array}{l}\text { Filtered } \mathrm{Na} \\
\text { excreted }\end{array}$ & $\frac{\mathrm{T}_{\mathrm{HCO}}}{\mathrm{GFR}}$ & $\mathrm{T}_{\mathrm{HCO}}$ \\
\hline & $m l / m i n$ & $m E q / l i t e r$ & $\mu E q / \min$ & $\mu E q / \min$ & $\mu E q / \min$ & $\%$ & $\begin{array}{c}m E q / \text { liter } \\
G F R\end{array}$ & $\mu E q / \min$ \\
\hline \multicolumn{9}{|c|}{ Inulin prime and sustain I $(5 \% \mathrm{D} / \mathrm{W}$ at $2.5 \mathrm{ml} / \mathrm{min})$ started at $-85 \mathrm{~min}$} \\
\hline 1 & 10.2 & 16.6 & 177.5 & 0.654 & 81.5 & 6.4 & 17.3 & 176.9 \\
\hline 2 & 10.2 & 15.4 & 165.0 & 0.675 & 112.0 & 8.8 & 16.1 & 164.3 \\
\hline 3 & 11.0 & 15.0 & 173.5 & 0.813 & 155.0 & 11.5 & 15.7 & 172.7 \\
\hline \multicolumn{9}{|c|}{ Sustain II $5 \%$ bicarbonate solution at $2.5 \mathrm{ml} / \mathrm{min}$} \\
\hline 4 & 11.5 & 16.6 & 200 & 1.3 & 149 & 10.5 & 17.3 & 198.7 \\
\hline 5 & 12.3 & 18.5 & 239 & 3.3 & 199 & 12.9 & 19.1 & 235.7 \\
\hline 6 & 12.9 & 21.4 & 290 & 4.4 & 173 & 10.5 & 22.1 & 285.7 \\
\hline 7 & 11.3 & 22.6 & 268 & 8.5 & 162 & 11.1 & 22.9 & 259.5 \\
\hline 8 & 12.9 & 24.0 & 326 & 17.8 & 189 & 11.2 & 23.8 & 308 \\
\hline 9 & 11.9 & 25.5 & 319 & 24.8 & 177 & 11.2 & 24.7 & 294 \\
\hline 10 & 11.9 & 27.5 & 344 & 45.6 & 212 & 13.2 & 25.0 & 298 \\
\hline 11 & 13.3 & 29.4 & 410 & 84.0 & 279 & 15.3 & 24.5 & 326 \\
\hline \multicolumn{9}{|c|}{ Sustain III $4 \%$ bicarbonate solution in isotonic saline at $6 \mathrm{ml} / \mathrm{min}$} \\
\hline 12 & 11.5 & 29.7 & 358 & 96.6 & 315 & 19.7 & 22.7 & 261 \\
\hline 13 & 12.2 & 31.4 & 403 & 147.0 & 375 & 22.0 & 21.0 & 256 \\
\hline 14 & 15.4 & 32.7 & 529 & 224.0 & 572 & 26.1 & 19.8 & 305 \\
\hline 15 & 14.4 & 34.9 & 530 & 245.0 & 569 & 27.1 & 19.8 & 285 \\
\hline
\end{tabular}

TABLE VII

A Bicarbonate Tritration Study in a Patient with Chronic Glomerulonephritis and the Nephrotic Syndrome

\begin{tabular}{|c|c|c|c|c|c|c|c|c|c|c|c|c|c|c|}
\hline \multirow{3}{*}{$\begin{array}{c}\text { Clearance } \\
\text { period }\end{array}$} & \multirow{3}{*}{$\frac{\text { Time }}{\min }$} & \multirow{3}{*}{$\frac{\text { GFR }}{m l / \min }$} & \multicolumn{3}{|c|}{ Plasma } & \multicolumn{3}{|c|}{ Urine } & \multirow{2}{*}{\multicolumn{2}{|c|}{$\mathrm{HCO}_{8}$ reabsorption }} & \multirow{2}{*}{\multicolumn{2}{|c|}{$\mathrm{HCO}_{3}$ excretion }} & \multirow{3}{*}{ 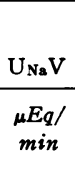 } & \multirow{3}{*}{$\begin{array}{c}\begin{array}{c}\text { Filtered } \\
\text { Na } \\
\text { excreted }\end{array} \\
\%\end{array}$} \\
\hline & & & \multirow[t]{2}{*}{$\mathrm{pH}$} & \multirow{2}{*}{$\frac{\mathrm{PCO}_{2}}{m m \mathrm{Hg}}$} & \multirow{2}{*}{$\frac{\mathrm{HCO}_{3}}{\begin{array}{c}m E q / \\
\text { liter }\end{array}}$} & \multirow[t]{2}{*}{$\mathrm{pH}$} & \multirow{2}{*}{$\frac{\mathrm{PCO}_{2}}{m m \mathrm{Hg}}$} & \multirow{2}{*}{$\frac{\mathrm{HCO}_{8}}{\begin{array}{c}m E q / \\
\text { liter }\end{array}}$} & & & & & & \\
\hline & & & & & & & & & $\begin{array}{c}\mu E q / \\
\min \end{array}$ & \multirow[t]{2}{*}{$\begin{array}{l}m E q / \\
\text { liter } \\
G F R\end{array}$} & \multirow[t]{2}{*}{$\begin{array}{c}\mu E q / \\
\min \end{array}$} & \multirow[t]{2}{*}{$\begin{array}{c}m E q / 100 \\
G F R\end{array}$} & & \\
\hline & Inulin pr & e and $s$ & stain I & $(5 \% \mathrm{D} / \mathrm{V}$ & $2.5 \mathrm{ml} / \mathrm{n}$ & a) star & ed at -8 & $\min$ & & & & & & \\
\hline 1 & $0-57$ & 6.80 & 7.36 & 30.3 & 16.7 & 4.80 & 34.1 & 0.039 & 119 & 17.5 & 0.05 & $<0.01$ & 15.0 & 1.83 \\
\hline 2 & $57-82$ & 7.45 & 7.37 & 31.2 & 18.5 & 4.75 & 32.2 & 0.034 & 145 & 19.4 & 0.04 & $<0.01$ & 15.0 & 1.56 \\
\hline \multirow[t]{2}{*}{3} & $82-109$ & 8.52 & 7.32 & 34.7 & 17.7 & 4.80 & 29.7 & 0.035 & 159 & 18.6 & 0.05 & $<0.01$ & 23.7 & 2.18 \\
\hline & \multicolumn{8}{|c|}{ Sustain II $5 \%$ bicarbonate solution at $2 \mathrm{ml} / \mathrm{min}$} & & & & & & \\
\hline 4 & $109-142$ & 7.40 & 7.34 & 34.8 & 18.3 & 4.80 & 32.7 & 0.039 & 142 & 19.2 & 0.05 & $<0.01$ & 20.0 & 2.11 \\
\hline 5 & $142-171$ & 6.84 & 7.38 & 35.5 & 20.4 & 4.90 & 34.0 & 0.050 & 146 & 21.4 & 0.07 & $<0.01$ & 17.7 & 2.03 \\
\hline 6 & $171-205$ & 6.73 & 7.43 & 34.6 & 22.0 & 4.90 & 33.0 & 0.049 & 156 & 23.1 & 0.06 & $<0.01$ & 15.5 & 1.78 \\
\hline 7 & $205-234$ & 8.10 & 7.47 & 34.5 & 24.1 & 4.90 & 36.0 & 0.053 & 203 & 25.3 & 0.07 & $<0.01$ & 14.8 & 1.43 \\
\hline 8 & $234-254$ & 8.55 & 7.44 & 33.6 & 22.3 & 5.05 & 34.9 & 0.073 & 226 & 23.4 & 0.09 & $<0.01$ & 14.3 & 1.26 \\
\hline 9 & $254-274$ & 8.47 & 7.51 & 30.5 & 23.7 & 5.00 & 33.6 & 0.063 & 211 & 24.9 & 0.07 & $<0.01$ & 13.6 & 1.24 \\
\hline 10 & $274-305$ & 7.60 & 7.49 & 36.0 & 26.8 & 5.00 & 34.6 & 0.065 & 214 & 28.1 & 0.07 & $<0.01$ & 12.5 & 1.24 \\
\hline 11 & $305-338$ & 7.55 & 7.49 & 36.2 & 26.5 & 5.10 & 33.7 & 0.079 & 211 & 27.8 & 0.09 & $<0.01$ & 13.7 & 1.33 \\
\hline 12 & $338-367$ & 7.79 & 7.55 & 34.5 & 29.4 & 5.19 & 35.5 & 0.103 & 241 & 30.9 & 0.12 & $<0.01$ & 13.2 & 1.24 \\
\hline 13 & $367-398$ & 7.50 & 7.54 & 35.5 & 29.7 & 5.31 & 37.6 & 0.144 & 234 & 31.2 & 0.18 & $<0.01$ & 14.0 & 1.40 \\
\hline 14 & $398-435$ & 5.91 & 7.58 & 34.6 & 31.0 & 5.80 & 40.7 & 0.484 & 192 & 32.5 & 0.54 & $<0.01$ & 14.4 & 1.77 \\
\hline 15 & $435-465$ & 8.85 & 7.52 & 41.2 & 32.3 & 6.30 & 43.1 & 1.650 & 298 & 33.6 & 2.17 & 0.03 & 18.4 & 1.57 \\
\hline 16 & $465-494$ & 6.57 & 7.50 & 41.7 & 31.4 & 6.51 & 45.5 & 2.780 & 214 & 32.5 & 3.36 & 0.05 & 16.9 & 2.01 \\
\hline 17 & $494-525$ & 6.16 & 7.58 & 38.4 & 34.5 & 6.70 & 48.1 & 4.600 & 219 & 35.4 & 5.53 & 0.09 & 20.4 & 2.44 \\
\hline 18 & $525-559$ & 8.17 & 7.57 & 38.4 & 34.4 & 6.95 & 49.0 & 8.300 & 286 & 35.1 & 9.65 & 0.12 & 21.0 & 1.89 \\
\hline
\end{tabular}


cal manifestations of uremia. The bicarbonate titration curve for this study is shown in Fig. 5.

In the second patient with the nephrotic syndrome, bicarbonate reabsorption was studied over a plasma bicarbonate range of $20.5-33.6 \mathrm{mEq} /$ liter. Values for reabsorption showed no tendency to stabilize, rising from $21.8 \mathrm{mEq} /$ liter GFR during the control periods to $34.2 \mathrm{mEq} /$ liter GFR at the peak level of plasma bicarbonate.

\section{DISCUSSION}

The traditional interpretation of the kinetics of renal bicarbonate reabsorption has been strongly influenced by studies using the bicarbonate titration technique. The prototype of the titration curve that has been recorded for normal man is shown in the triangles in Fig. 1. As the plasma bicarbonate concentration is increased progressively, bicarbonate is completely reabsorbed until plasma levels approximate $26 \mathrm{mEq} /$ liter. At this concentration, which is the so-called bicarbonate threshold, bicarbonate begins to enter the urine. As plasma levels are increased further, the rate of reabsorption tends to become constant such that approximately $2.8 \mathrm{mEq}$ of bicarbonate are reabsorbed per $100 \mathrm{ml}$ of glomerular filtrate $(28 \mathrm{mEq} /$ liter GFR). This apparent maximum velocity of reabsorption has been accepted for many years as the normal $\mathrm{Tm}$ for bicarbonate. However, there are reasons to suspect that the classic titration curve may be influenced by the condition of the titration experiment, which of necessity involves marked expansion of the extracellular fluid volume. Volume expansion is known to diminish fractional sodium reabsorption in the proximal tubule $(8,9)$ and thus to influence the setting of glomerulotubular balance for sodium. On a priori grounds, volume expansion might be expected to influence sodium-hydrogen exchange in the proximal tubules as well as sodium reabsorbed with chloride; thus the status of extracellular fluid volume could also influence the setting of glomerulotubular balance for bicarbonate. Recent observations by Purkerson, Lubowitz, White, and Bricker, in the rat, lend support to the latter possibility (4). When the bicarbonate titration procedure was modified so as to minimize ECF volume expansion, no $\mathrm{Tm}$ could be demonstrated in normal rats in which plasma bicarbonate concentrations were elevated to levels as high as $60 \mathrm{mEq} /$ liter. However, when volume expansion was exaggerated, a clear tendency for reabsorption to plateau was observed and the threshold for bicarbonate excretion was decreased. ECF volume expansion also has been shown to decrease bicarbonate reabsorption in individual superficial nephrons of the rat (10).

A second state characterized by increased fractional sodium excretion and natriuresis per nephron is nephron

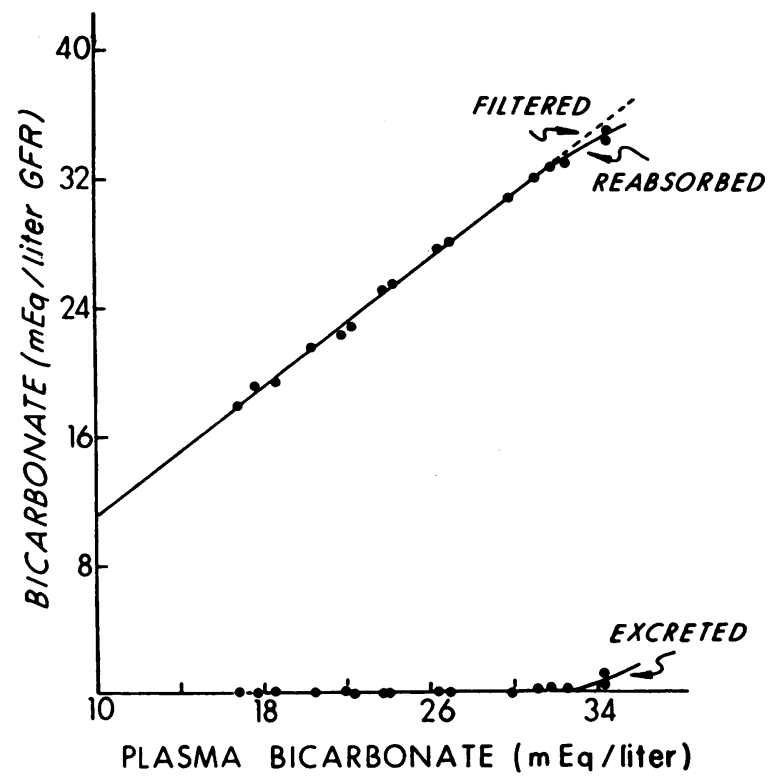

FIGURE 5 Bicarbonate titration curve in a nephrotic patient with chronic glomerulonephritis and a GFR of $7 \mathrm{ml} / \mathrm{min}$.

reduction with a decreased GFR. Indeed, in advanced renal disease the maintenance of sodium balance on an average salt intake requires the excretion of a substantially greater fraction of filtered sodium than is encountered in saline diuresis in subjects with normal renal function (11). It is of interest, therefore, that reduction of the nephron population by approximately $75 \%$ in rats initiated alterations in the bicarbonate titration curve comparable to those observed after exaggerated expansion of ECF (5). Furthermore, micropuncture studies have shown that nephron reduction in the rat leads to marked inhibition of fractional bicarbonate reabsorption in individual superficial nephrons of the rat. ${ }^{1}$

The present studies were performed to examine the influence of both extracellular fluid volume expansion and uremia on bicarbonate reabsorption in man. $\mathrm{Pa}$ tients were studied with a range of glomerular filtration rates from $170 \mathrm{ml} / \mathrm{min}$ to $2 \mathrm{ml} / \mathrm{min}$. The normal subjects and the patients with renal disease in whom GFR was greater than $30 \mathrm{ml} / \mathrm{min}$ fell into one group. When ECF volume expansion was minimized by salt deprivation and diuretic administration in advance of the titration study and by the infusion of the minimum amount of sodium bicarbonate consistent with the desired elevation of plasma bicarbonate concentrations during the study, no definite $\mathrm{Tm}$ for bicarbonate was observed. In four of these five subjects the excretion

${ }^{1}$ Lubowitz, H., M. L. Purkerson, and N. S. Bricker. 1969. A micropuncture study of the effect of nephron reduction on proximal tubular bicarbonate reabsorption in the rat. In press. 
rate of sodium during the control clearance periods was less than $0.1 \%$ of the filtered load.

In the same group of subjects, exaggerated expansion of ECF led to an alteration in the patterns of bicarbonate reabsorption. In comparison to the minimized expansion studies, the threshold for bicarbonate excretion was lower and there was a tendency for bicarbonate reabsorption to stabilize below $30 \mathrm{mEq} /$ liter of GFR. These subjects exhibited a moderate degree of natriuresis before the onset of the bicarbonate infusion and the natriuresis became quite marked during the course of the studies.

The patients with glomerular filtration rates of 20 $\mathrm{ml} / \mathrm{min}$ or less resembled the exaggerated expansion group although ECF volume expansion was not exaggerated. However, in order to maintain sodium balance at a GFR of $20 \mathrm{ml} / \mathrm{min}$ on a $4 \mathrm{~g}$ salt intake, approximately $2 \%$ of the filtered sodium must be excreted and the lower the GFR the greater must this percentage be (e.g. at a GFR of $5 \mathrm{ml} / \mathrm{min}$, approximately $10 \%$ of the filtered sodium must be excreted). It is of interest, therefore, that the patients with the more markedly reduced levels of GFR and the higher fractional sodium excretion rates reabsorbed bicarbonate in a manner qualitatively similar to the subjects with higher GFR's subjected to exaggerated expansion of ECF volume. It also is of interest that the lower the level of GFR, and thus the greater the rate of fractional sodium excretion, the more striking was the alteration of bicarbonate reabsorption (see Fig. 4). Finally, when acute volume expansion was induced in uremic patients with stable values for bicarbonate reabsorption per unit of GFR, fractional sodium excretion increased markedly, the apparent $\mathrm{Tm}$ for bicarbonate was perturbed, and bicarbonate reabsorptive rates, both per unit of GFR and in absolute terms, diminished.

Additional evidence relating fractional sodium reabsorption to the patterns of bicarbonate reabsorption emerges from the studies on the two nephrotic patients. These two subjects, despite very low glomerular filtration rates, were edema formers and thus did not exhibit the natriuresis per nephron which is characteristic of patients with their levels of GFR. Of considerable interest is the fact that their bicarbonate titration curves were strikingly different from other patients with uremia, resembling those for the nonuremic subjects studied during minimized ECF volume expansion. Indeed, the titration curves in the two uremic nephrotic patients exhibited the highest slope for bicarbonate reabsorption per unit of GFR vs. plasma bicarbonate concentration of all the subjects studied.

Several possible conclusions emerge from these studies. (a) The "normal" $\mathrm{Tm}$ for bicarbonate in man may actually represent an inhibited rate of bicarbonate reabsorption imposed by volume expansion attendant upon the standard titration protocol.

(b) Intrinsic renal disease per se does not appear to depress bicarbonate reabsorption per unit of GFR in the residual nephrons. The evidence for this is found in the titration curves of patients with GFR levels of over $30 \mathrm{ml} / \mathrm{min}$ during minimized ECF volume expansion and in the two uremic patients who were nephrotic. In both groups, bicarbonate reabsorption increased over the entire range of plasma bicarbonate levels employed and threshold values were higher than the usually accepted normal.

(c) The intensity of natriuretic forces appears to influence the character of the bicarbonate titration curve. In both of the nephrotic uremic patients and in four of the five subjects studied during minimized expansion, fractional sodium excretion tended to remain below $2 \%$ even at the highest plasma bicarbonate concentrations obtained. On the other hand, in the presence of exaggerated ECF expansion and in the non-nephrotic uremic patients peak values for fractional sodium excretion ranged from $5 \%$ to $40 \%$. Moreover, the higher the fractional sodium excretion rate, the lower was the apparent $\mathrm{Tm}$ for bicarbonate. Finally, when the uremic patients with stable rates of bicarbonate reabsorption were subjected to acute volume expansion, natriuresis increased strikingly and bicarbonate reabsorption decreased correspondingly.

Thus bicarbonate reabsorption appears to be influenced by the concurrent activity of the factors controlling sodium excretion. In all probability, therefore, the factors that modulate sodium reabsorption in the proximal convoluted tubule must have a secondary influence on bicarbonate reabsorption whether the latter occurs in consequence of sodium-hydrogen exchange or the reabsorption of bicarbonate as an ion.

One final possibility must be considered in explanation for the present data. Both volume expansion and nephron reduction may be attended by an increase in GFR per nephron especially in the superficial nephrons $(12,13)$. The effects of either acute or chronic hyperfiltration on the kinetics of bicarbonate reabsorption remain to be established and although the experimental induction of hyperfiltration both in normal dogs (14) and uremic man (11) does not influence fractional sodium excretion, the possibility was considered that the decrease in bicarbonate reabsorption expressed per unit of GFR seen with exaggerated ECF volume expansion was secondary to an increase in GFR per nephron rather than to inhibition of bicarbonate reabsorption. However, the opportunity to compare absolute rates of bicarbonate reabsorption at comparable levels of GFR 
and comparable plasma bicarbonate concentrations existed in three of the subjects studied during both minimized and exaggerated expansion. The data presented in Table $\mathrm{V}$ suggest that the exaggerated expansion was accompanied not only by a decrease in fractional bicarbonate reabsorption but by a decrease in absolute bicarbonate reabsorption. The same phenomenon was observed in the uremic patients in whom ECF volume expansion was exaggerated after an apparent $\mathrm{Tm}$ for bicarbonate had been reached (Table VI). Thus volume expansion does appear to inhibit tubular reabsorption of bicarbonate.

\section{ACKNOWLEDGMENTS}

This work was supported by U. S. Public Health Service Research Grant AM-09975 and Grant FR-36, General Clinical Research Centers Branch, Division of Research Facilities and Resources. Dr. Bricker is a recipient of a U. S. Public Health Service Research Career Award.

\section{REFERENCES}

1. Berliner, R. W. 1963. Outline of renal physiology. In Diseases of the Kidney. M. B. Strauss and L. G. Welt, editors. Little, Brown \& Co., Boston. 30.

2. Pitts, R. F. 1968. Physiology of the kidney and body fluids. Year Book Medical Publishers, Chicago.

3. Roberts, K. E., H. T. Randall, P. Vanamee, and J. W. Poppell. 1956. Renal mechanisms involved in bicarbonate absorption. Metab. (Clin. Exp.). 5: 404.

4. Purkerson, M. L., H. Lubowitz, R. W. White, and N. S. Bricker. 1969. On the influence of extracellular fluid volume expansion on bicarbonate reabsorption in the rat. J. Clin. Invest. 48: 1754.
5. Purkerson, M. L., H. Lubowitz, and N. S. Bricker. 1967. The genesis of the bicarbonate leak in chronic renal disease. Clin. Res. 15: 367

6. Slatopolsky, E., and A. M. Robson, I. Elkan, and N. S. Bricker. 1968. Control of phosphate excretion in uremic man. J. Clin. Invest. 47: 1865.

7. Roe, J. H., J. H. Epstein, and N. P. Goldstein. 1949. A photometric method for the determination of inulin in plasma and urine. J. Biol. Chem. 178: 839.

8. Dirks, J. H., W. J. Cirksena, and R. W. Berliner. 1965. The effect of saline infusion on sodium reabsorption by the proximal tubule of the dog. J. Clin. Invest. 44: 1160 .

9. Cortney, M. A., M. Myelle, W. E. Lassiter, and C. W. Gottschalk. 1965. Renal tubular transport of water, solute and PAH in rats loaded with isotonic saline. Amer. J. Physiol. 209: 1199.

10. Kunau, R., A. Frick, F. C. Rector, Jr., and D. W. Seldin. 1966. Effect of extracellular fluid (ECF) volume expansion, $\mathrm{K}^{+}$deficiency and $\mathrm{pCO}_{2}$ on bicarbonate reabsorption in the rat. Clin. Res. 14: 380.

11. Slatopolsky, E., I. O. Elkan, C. Weerts, and N. S. Bricker. 1968. Studies on the characteristics of the control system governing sodium excretion in uremic man. J. Clin. Invest. 47: 521 .

12. Stein, J. H., L. J. Barton, H. Mandin, L. H. Lackner, F. C. Rector, Jr. and D. W. Seldin. 1969. Effect of extracellular volume expansion on proximal tubular sodium reabsorption and distribution of renal blood flow and glomerular filtrate in the dog. Clin. Res. 17: 449.

13. Lubowitz, H., M. L. Purkerson, M. Sugita, and N. S. Bricker. 1969. GFR per nephron and per kidney in chronically diseased (pyelonephritic) kidney of the rat. Amer. J. Physiol. 217: 853.

14. Lindheimer, M. D., R. C. Lalone, and N. G. Levinsky. 1967. Evidence that an acute increase in glomerular filtration has little effect on sodium excretion in the dog unless extracellular volume is expanded. J. Clin. Invest. 46: 256 